\title{
Efficient watershed modeling using a multi-site weather generator for meteorological data
}

\author{
M. Khalili, R. Leconte \& F. Brissette \\ Department of Construction Engineering, \\ École de Technologie Supérieure, Montréal, Québec, Canada
}

\begin{abstract}
The multi-site generation of precipitation data is developed using a Richardson (1981) WGEN-type weather generator. This approach is based on spatial autocorrelation to analyze patterns in space and investigate the dependence of weather data at multiple locations. Reproducing the dependence between meteorological data at several stations should make the hydrological model results more realistic. The Chute du diable watershed and surrounding area located in the province of Quebec, Canada was used to test the proposed approach. Daily spatial autocorrelations between precipitation occurrences and amounts were successfully reproduced as well as total monthly precipitation and monthly numbers of rainy days. A hydrological model has been used to quantify the natural inflow process. As envisaged, the multi-site generation of weather data produced more practical natural inflow hydrographs, compared to those obtained using a uni-site weather generator.
\end{abstract}

Keywords: weather generator, precipitation, Markov chain, spatial autocorrelation, hydrological modeling.

\section{Introduction}

Weather generators can be used to generate climatic data (precipitation, temperature, solar radiation...) with the same statistical properties as the observed ones. Most weather generators operate for a single site, e.g. [15], [2] and [7]. Therefore, they ignore the regional coherence and the spatial dependence between the stations, which entail many problems in the hydrological modeling results, obtained using the simulated time series of meteorological data. 
A few models were developed for multi-site simulation of weather variables, in particular for daily precipitations such as space-time model of Bardossy and Plate [1], the non-homogeneous hidden Markov model of Bellone et al. [3] and Hughes et al. [11], the nearest-neighbor resampling approach developed by Buishand and Brandsma [5], and a method based on serially independent but spatially correlated random numbers developed by Wilks [16].

A regionalization approach based on spatial autocorrelation is proposed to improve the watershed modeling. It is applied to the Chute du diable watershed and surrounding area located in the province of Quebec, Canada. This technique performed successfully in simulating meteorological data and runoff process. The proposed methodology is presented in section 2 of this article. Section 3 describes the results obtained in the studied basin.

\section{Methodology}

\subsection{Uni-site weather generator}

Following the Richardson approach [13], a uni-site weather generator uses a first-order two states Markov chain to simulate daily precipitation occurrence $X_{t}(k)$ at site $\mathrm{k}$ on day t. A uniform $[0,1]$ random number $u_{t}(k)$ is compared with a critical probability, which is equal to one of the transition probabilities depending on the state of the previous day:

$$
p_{c}(k)=\left\{\begin{array}{l}
p_{01}(k), \text { if } X_{t-1}(k)=0 \\
p_{11}(k), \text { if } X_{t-1}(k)=1
\end{array} .\right.
$$

A wet day is simulated if the random number is smaller than this critical probability:

$$
X_{t}(k)=\left\{\begin{array}{l}
1, \text { if } u_{t}(k) \leq p_{c}(k) \\
0, \text { otherwise }
\end{array} .\right.
$$

Another uniform $[0,1]$ random number $v_{t}(k)$ is used to simulate the synthetic precipitation amounts by the inversion of the distribution function of amounts. In the case of an exponential distribution [14], the precipitation amounts $r_{t}(k)$ can be computed as:

$$
r_{t}(k)=-\ln \left(1-v_{t}(k)\right) / \lambda(k)
$$

where $\lambda$ is the parameter describing the exponential distribution function:

$$
f[r(k)]=1-\exp (-\lambda r(k)) .
$$




\subsection{Multi-site weather generator}

The Multi-site weather generator used here is based on the concept of spatial autocorrelation. This theory has been used in a wide array of applications in which the spatial dependence has to be accounted for, such as social, economic and physics sciences. Spatial autocorrelation is the correlation between values of a single variable in geographic space. The analogous statistic to spatial autocorrelation is serial autocorrelation which is the correlation between values of a single variable at different time. Spatial autocorrelation can be measured by statistical indicators such as Moran's I $[10,12]$.

$$
I=\frac{\sum_{i=1}^{n}\left(x_{i}-\bar{x}\right) \sum_{j=1}^{n} w_{i j}\left(x_{j}-\bar{x}\right) / \sum_{i=1}^{n} \sum_{j=1}^{n} w_{i j}}{\sqrt{\sum_{i=1}^{n}\left(x_{i}-\bar{x}\right)^{2} / n} \sqrt{\sum_{i=1}^{n}\left(x_{i}-\bar{x}\right)^{2} / n}} .
$$

where $x_{i}$ denotes the observed value at location $\mathrm{i}, \bar{x}$ is the average of the $x_{i}$ over the $\mathrm{n}$ locations and $w_{i j}$ is the spatial weight between two locations $\mathrm{i}$ and $\mathrm{j}$. The matrix form of Moran's I contains a spatial weight matrix whose elements are the weights $w_{i j}$. Generally, these weights are in a row-standardized form, which means that all weights in a row sum to 1 and by convention $w_{i i}=0$. Moran's I takes values greater than zero if the geographically nearby observations are similar, lower than zero if they are dissimilar and equal to zero if these observations are independent.

The weather generator is modified to simulate precipitation data with daily spatial autocorrelations, measured by Moran's I, identical to those observed. Indeed, the random numbers used in the weather generator, eqns. (2) and (3), are transformed to spatially autocorrelated ones whose spatial autocorrelations will reproduce the spatial autocorrelations computed between observed precipitation series. To generate spatially autocorrelated random numbers, a spatial moving average process $[6,8]$ is used:

$$
V=\gamma \times W \times u+u .
$$

where $V(n, 1)$ is a vector of $\mathrm{n}$ spatially autocorrelated random numbers to be used for $\mathrm{n}$ locations.

$W(n, n)$ is a weights matrix.

$u(n, 1)$ is a vector of $\mathrm{n}$ independent and uniformly distributed random variables. $\gamma$ is the moving average coefficient.

Different $\gamma$ values provide random numbers with different spatial autocorrelations and accordingly simulated precipitation processes exhibit 
different daily spatial autocorrelations. Therefore, a relationship between the coefficients $\gamma$ and the spatial autocorrelations of precipitation occurrences and amounts can be used to identify the particulars $\gamma$, which yield the set of random numbers reproducing the observed daily spatial autocorrelations of precipitation occurrences and amounts. These meteorological data will be then used as input into the hydrological model.

\subsection{Hydrological modeling}

The HSAMI model [4] is used for hydrological modeling. HSAMI is a lumped conceptual model consisting of three linear reservoirs in cascades currently used by Hydro-Québec to forecast and to simulate natural inflows to reservoirs or runoff at watershed outlets. A set of meteorological data consisting of rainfall, snow, minimal and maximal temperature and insulation [9] is required to simulate the hydrological processes. Fig. 1 gives a simplified diagram of HSAMI [9].

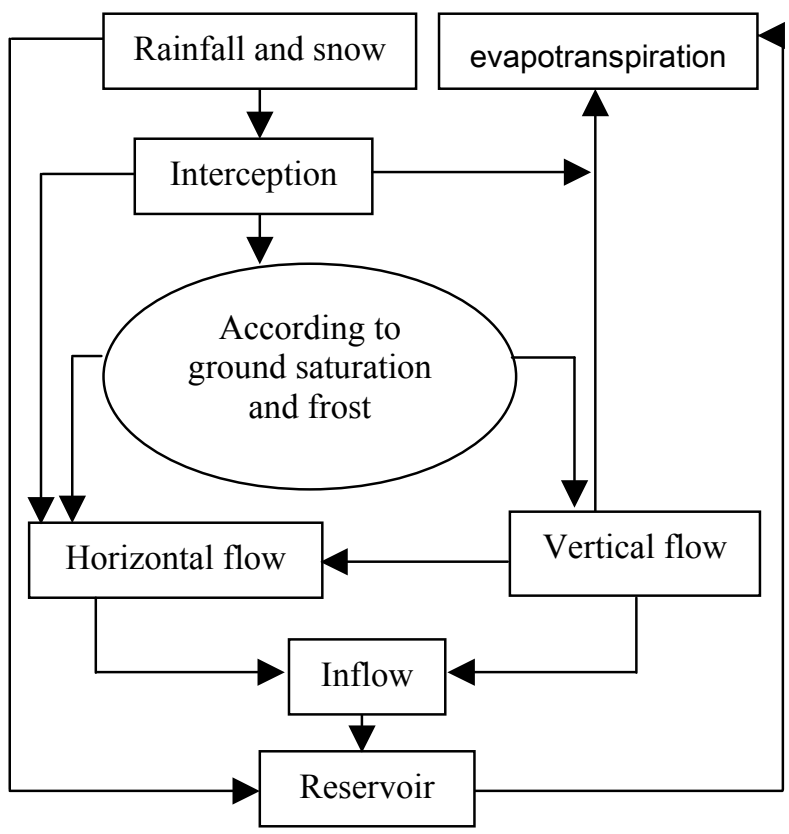

Figure 1: $\quad$ Simplified diagram of HSAMI [9].

\section{Results and discussion}

Seven weather stations were used in Chute du diable watershed and surrounding area in the province Quebec of Canada (fig. 2): 1: Péribonca, 2: Normandin CDA, 3: Hémon, 4: Bonnard, 5: Chute du diable, 6: Chute des passes, 7: St- 
Leon-de-Labrecque. The uni-site and multi-site approaches described above were used to simulate precipitation data at these stations to be incorporated in the hydrological model. The uni-site approach simulates the data at each weather station independently from the others. Thus, the spatial dependence in precipitation data is ignored. The multi-site approach produces daily spatial autocorrelations that are identical to those observed at the stations.

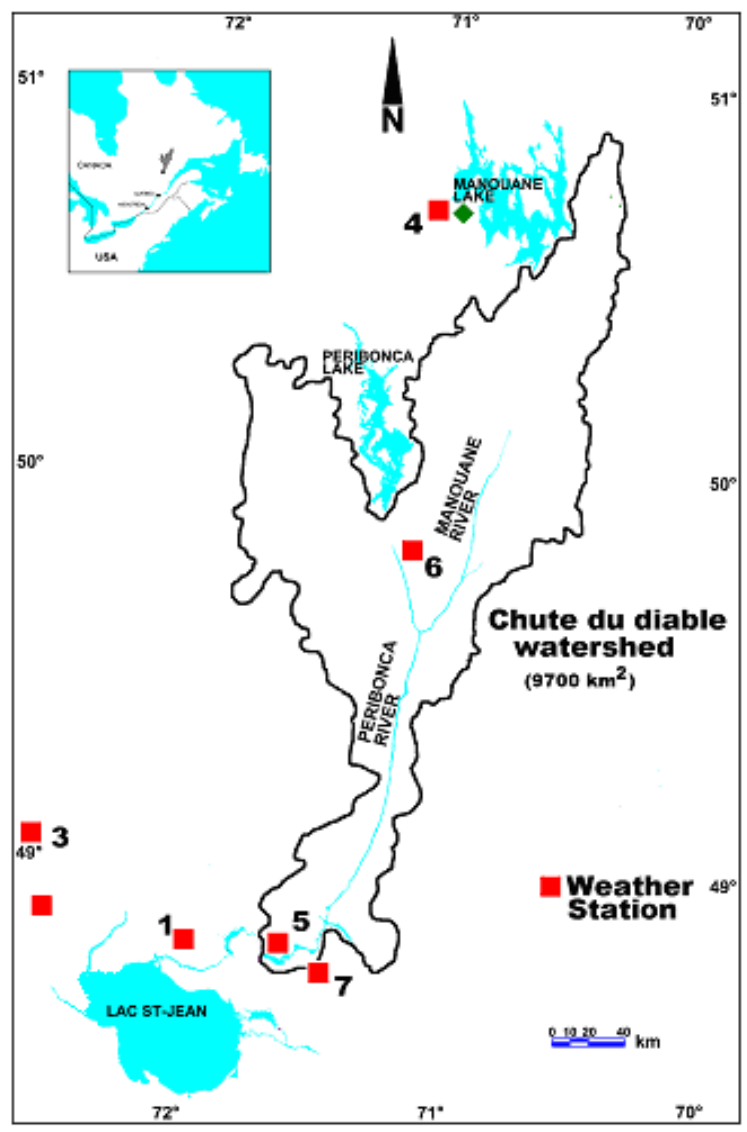

Figure 2: $\quad$ Chute du Diable watershed with locations of weather stations.

Fig. 3 gives an example of observed versus simulated monthly numbers of rainy days at all stations for the month of March and fig. 4 gives the total monthly precipitation at all stations for the month of September. Similar results were obtained for all the other months. These curves show a good agreement between the observed and simulated precipitation occurrences and amounts. Furthermore, daily spatial autocorrelations of precipitation occurrences and amounts were well reproduced, as shown by fig. 5 and 6 , which illustrate these results for March and September respectively. Again, similar results were obtained for the remaining months. 


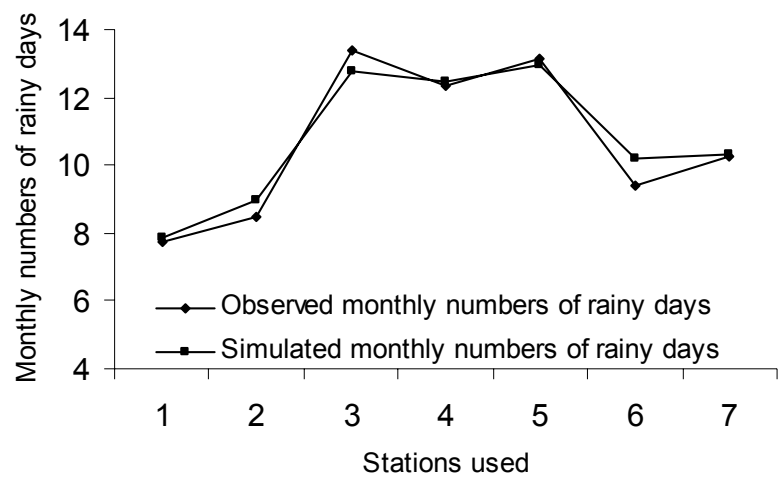

Figure 3: Observed versus simulated monthly numbers of rainy days at the seven stations for March.

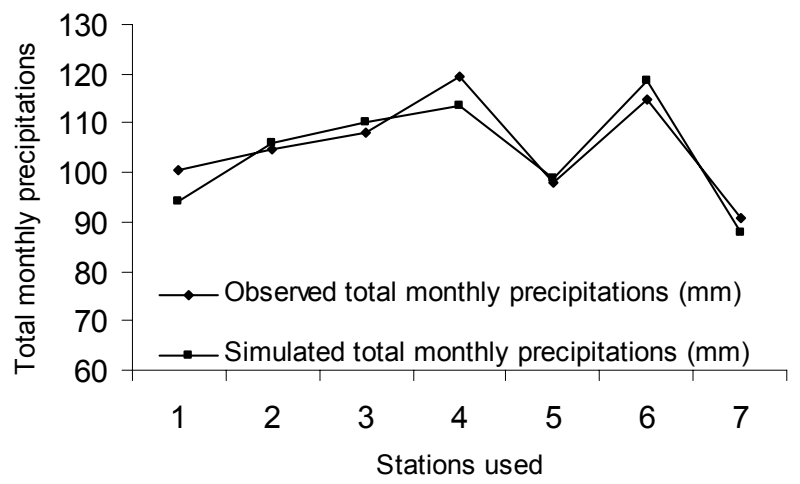

Figure 4: Observed and simulated Total monthly precipitations at the seven stations for September.

HSAMI hydrological model requires the mean precipitation over the studied area. Five weather stations were then selected within and around Chute du diable watershed according to their proximity to the watershed (stations 1, 4, 5, 6 and 7). An interpolation of precipitation values over this area is required to compute the mean precipitation using the Thiessen polygons. This operation was done using the precipitation data simulated by the uni-site and multi-site approaches. However, the temperature data were simulated only by the uni-site approach, as a multi-site model of temperature data is currently under development. The meteorological data were then incorporated in the HSAMI hydrological model.

Five years of meteorological and natural inflow data were used for calibrating the HSAMI model. Natural inflows were then simulated using the two types of precipitation data. The simulation results from the input precipitation data generated using the multi-site approach were found to be more realistic than 
using the input precipitation data generated from the uni-site approach. Typically, the simulated inflow hydrograph from the multi-site approach displays the spring as well as the late summer and early fall floods (AugustOctober), while the simulated hydrograph from the uni-site approach indicates only the spring floods, see fig. 7 .

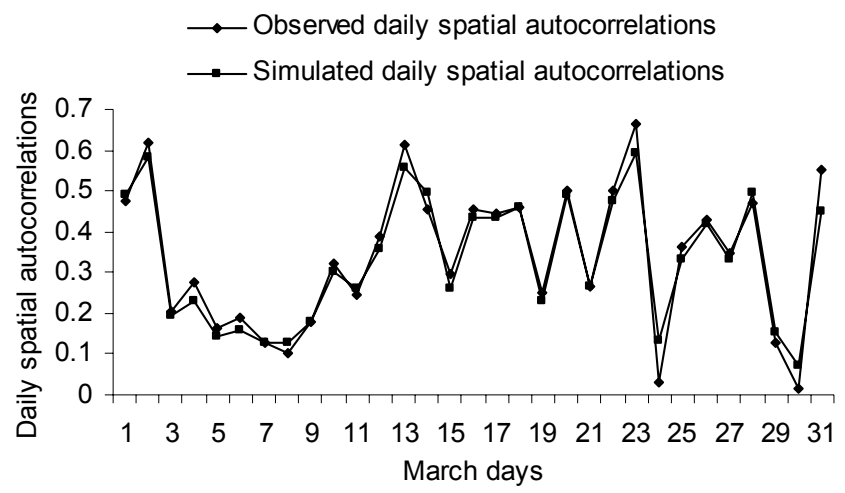

Figure 5: Observed and simulated daily spatial autocorrelations of precipitation occurrences for March.

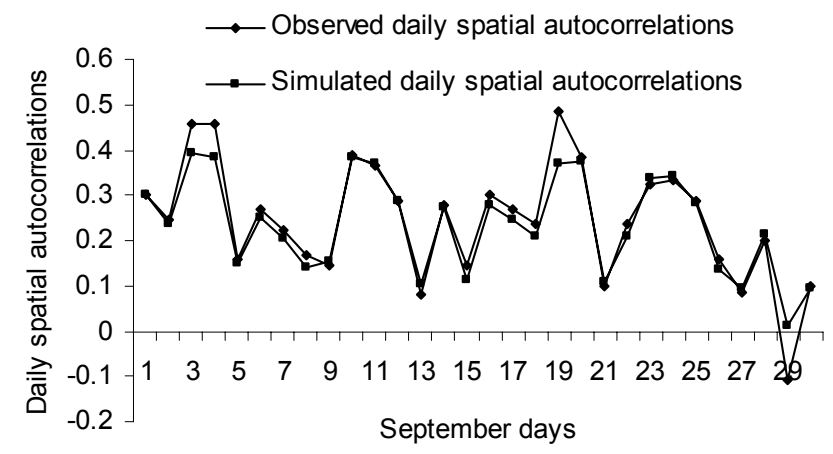

Figure 6: Observed and simulated daily spatial autocorrelations of precipitation amounts for September.

The hydrological model fed with multi-site generated precipitation data was able to better mimic the observed process because of spatial dependence of rainfall and snowfall, which was reproduced between the multiple sites in spite of the lumped nature of the HSAMI. In reality, the climate responsible of the precipitation process extends at the regional scale rather than at the station location and constrains the observations in a given station to be correlated to those in nearby area. Table 1 shows how the multi-site approach improves the 
simulation of extreme events. Indeed, the extreme natural inflows from the multi-site approach (Multi) were more consistent with the observed ones (Obs) while those from the uni-site approach (Uni) were systematically underestimated.

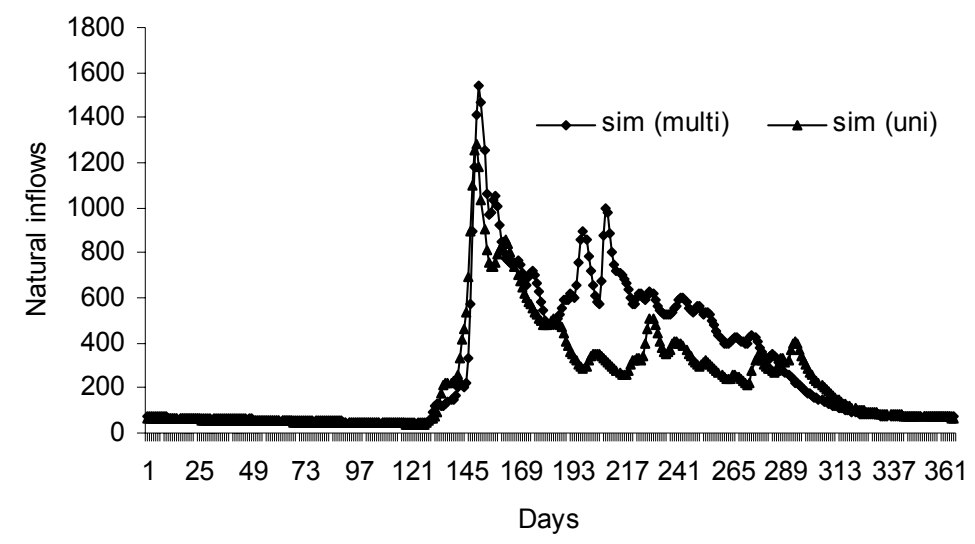

Figure 7: $\quad$ Example for Multisite and unisite simulated natural inflows.

Table 1: $\quad$ Observed and simulated extreme events by the two approaches.

\begin{tabular}{|c|c|c|c|}
\cline { 2 - 4 } \multicolumn{1}{c|}{} & \multicolumn{3}{c|}{ Natural inflows $\left(\mathrm{m}^{3} / \mathrm{s}\right)$} \\
\hline Return periods & Obs & Multi & Uni \\
\hline 2 & 1243.5 & 1321.45 & 1022.45 \\
\hline 5 & 1419 & 1315.1 & 1211.2 \\
\hline 10 & 1729 & 1813.55 & 1339.3 \\
\hline
\end{tabular}

\section{Conclusion}

The Chute du diable watershed and surrounding area located in the province of Quebec, Canada is used to investigate the behaviour of hydrological modeling using uni-site and multi-site generation approaches for precipitation data. The multi-site approach performed successfully in simulating both precipitation occurrences and amounts. This result impacts on the hydrological modeling, as demonstrated using the HSAMI model, which better displayed the late summerearly fall flood when multi-site approach was used. Other performance criteria are under investigation to further demonstrate the potential and capabilities of coupling a multi-site precipitation model to a hydrological model.

\section{References}

[1] Bardossy, A. \& Plate, E.J., Space-time model for daily rainfall using atmospheric circulation patterns. Water resources research, 28, pp. 1247$1259,1992$. 
[2] Bardossy, A. \& Plate, E.J., Modeling daily rainfall using a semi-Markov representation of circulation pattern occurrence. Journal of Hydrology, 122, pp. 33-47, 1991.

[3] Bellone, E., Hughes, J.P. \& Guttorp, P., A hidden Markov model for downscaling synoptic atmospheric patterns to precipitation amounts. Climate Research, 15, pp. 1-12, 2000.

[4] Bisson, J.L. \& Roberge, F., Prévision des apports naturels: Expérience d'Hydro-Québec. Compte-rendu de l'Atelier sur la prévision du débit, Toronto, novembre 1983.

[5] Buishand, T.A. \& Brandsma, T., Multisite simulation of daily precipitation and temperature in the Rhine basin by nearest-neighbour resampling. Water resources research, 37(11), pp. 2761-2776, 2001.

[6] Cliff, A.D. \& Ord, J.K., Spatial processes: Models and applications. London: Pion, 1981.

[7] Corte-Real, J., Xu, H. \& Qian, B. A weather generator for obtaining daily precipitation scenarios based on circulation patterns. Climate Research, 13, pp. 61-75, 1999.

[8] Cressie, N.A.C., Statistics for spatial data. Wiley series in probability and mathematical statistics, John Wiley \& Sons, 900 p, 1993.

[9] Fortin, V., Le modèle météo-apport HSAMI: historique, théorie et application. Rapport de recherche, révision 1,5. Institut de recherche d'Hydro-Québec (IREQ), Varennes, Qué. 68 p, 2000.

[10] Griffith, D.A., Spatial autocorrelation and spatial filtering: Gaining understanding through theory and scientific visualization. Advances in spatial science, Springer, 247 p, 2003.

[11] Hughes, J.P., Guttorp, P. \& Charles, S., A nonhomogeneous hidden Markov model for precipitation occurrence. Applied statistics, 48, pp. 15 30, 1999.

[12] Odland. J., Spatial autocorrelation. Sage Publications: Newbury Park, 87 p, 1988.

[13] Richardson, C.W., Stochastic simulation of daily precipitation, temperature, and solar radiation. Water resources research, 17(1), pp. 182-190, 1981.

[14] Todorovic, P. \& Woolhiser, D.A., Stochastic model of daily rainfall. Proc. the symposium on statistical hydrology. Misc. Publ. 1275, U. S. D. A. Washington, Dc., pp. 232-246, 1974.

[15] Wilks, D.S. Conditioning stochastic daily precipitation models on total monthly precipitation. Water resources research, 25, pp. 1429-1439, 1989.

[16] Wilks, D.S., Multisite generalization of a daily stochastic precipitation generation model. Journal of Hydrology, 210, pp. 178-191, 1998. 